\title{
The relationship between talent identification testing parameters and performance in elite junior swimmers
}

\author{
Lachlan J.G. Mitchell ${ }^{\mathrm{a}, \mathrm{b}, \mathrm{d}, *}$, Ben Rattray ${ }^{\mathrm{b}, \mathrm{c}}$, Philo U. Saunders ${ }^{\mathrm{a}, \mathrm{b}}$, David B. Pyne ${ }^{\mathrm{a}, \mathrm{b}}$ \\ a Australian Institute of Sport, Australia \\ ${ }^{\mathrm{b}}$ University of Canberra Research Institute for Sport and Exercise, Australia \\ ' Discipline of Sport and Exercise Science, Faculty of Health, University of Canberra, Australia \\ d Queensland Academy of Sport, Australia
}

\section{A R T I C L E I N F O}

\section{Article history:}

Received 24 February 2018

Received in revised form 24 April 2018

Accepted 8 May 2018

Available online $\mathrm{xxx}$

\section{Keywords:}

Adolescent athlete

Growth

Puberty

Anaerobic capacity

Event specialisation

\begin{abstract}
A B S T R A C T
Objectives: In elite age-group swimming it is unclear to what degree common assessments of anthropometric, jump performance and front-crawl critical speed (CS) correlate with competition performance. Design: Cross-sectional field study.

Methods: Forty eight elite national-level junior swimmers (22 males, age $16.5 \pm 1.2$ y, 26 females, age $15.5 \pm 1.1 \mathrm{y}$; mean \pm SD) completed anthropometry tests, loaded and unloaded countermovement jumps and a series of front-crawl time-trials to determine CS and supra-CS distance capacity (D'). Years from peak height velocity (PHV) predicted from anthropometric data was used as a maturity indicator. Race performances within 3 months of testing were standardised to compare across distances and strokes. Multiple linear regression models were formulated using these data.

Results: Loaded jump height, mass, D', PHV and humerus breadth best predicted $100 \mathrm{~m}$ performance in males $\left(R^{2}{ }_{\text {Adj }}=0.88, p<0.001\right)$, while loaded jump height, chest depth and sitting height predicted female $100 \mathrm{~m}$ performances $\left(\mathrm{R}^{2}\right.$ Adj $\left.=0.74, \mathrm{p}=0.002\right)$. Loaded and unloaded jump height, mass, CS and PHV $\left(R^{2}\right.$ Adj $\left.=0.73, p=0.003\right)$ and $C S$ and chest depth $\left(R^{2}\right.$ Adj $\left.=0.33, p=0.03\right)$ predicted $200 \mathrm{~m}$ performance in males and females respectively.

Conclusions: Common assessments of power and aerobic capacity in elite junior swimmers explain more variance in competition performance for male than female swimmers, as well as for $100 \mathrm{~m}$ rather than $200 \mathrm{~m}$ events. These findings highlight the need to empirically assess testing regimens and suggest new tests in this population may be required.
\end{abstract}

(c) 2018 Sports Medicine Australia. Published by Elsevier Ltd. All rights reserved.

\section{Introduction}

Modern elite swimmers undergo many different laboratoryand pool-based tests to assess their physical development. Testing methods are well established at the senior level ${ }^{1}$ and are used to assess athlete ${ }^{2}$ progression and for talent identification (TID). Monitoring of elite swimmers typically focuses on characteristics related to the biomechanical, physiological and psychological determinants of performance. ${ }^{3}$ Aspects of each of the three major determinants are measured to explain variation in performance, both within an individual and to distinguish between competitors. The predictive success of a measured parameter may depend

Abbreviations: CS, critical speed; D', supra-critical speed distance capacity; PHV, peak height velocity.

* Corresponding author.

E-mail address: lachlan.mitchell@npsr.qld.gov.au (L.J.G. Mitchell). partly on its interaction with other characteristics, and partly on the competition requirements.

The event a swimmer is competing in can substantially influence the way a physical characteristic affects their performance. Selected anthropometric characteristics vary in swimmers competing in different distances and strokes, indicating there may be event-specific anthropometric requirements and events to which some swimmers may be better suited. ${ }^{4}$ A single cohort study in adolescent, national-level swimmers indicated that anthropometric variables correlated moderately with performance $(r=0.30-0.72) .^{5}$ In this investigation, anthropometric variables correlated more strongly with 200 IM performance than $400 \mathrm{~m}$ front-crawl performance. Event distance also seems likely to influence which variables are the most important between competitors. The effects of physiological characteristics are modulated by event distance; including both swimming-specific parameters, such as speed at lactate threshold, and general parameters such as $\dot{\mathrm{VO}}_{2}$ max and counter- 
movement jump (CMJ) performance. Speed at lactate threshold ${ }^{5}$ and $\dot{V O}_{2}$ max $^{6}$ are both more important as events become longer, an expected result given longer events require more aerobic energy. ${ }^{7,8}$ Countermovement jump performance is more closely aligned to performances over shorter distances, ${ }^{5,6}$ an effect that is likely driven by the increased influence of the $\operatorname{start}^{9}$ on shorter duration events. ${ }^{10}$ It seems that the selection of testing metrics should be specific to event distance to maximise the translation to competition performance.

While the energetic demands of competition are important, they are not the only variables that need to be considered when selecting monitoring parameters. Age, sex, maturity, ${ }^{11}$ fitness and recent training history all contribute as well. The selection of assessments is an important process to ensure the time efficiency and effectiveness of testing procedures for coaches, swimmers and sport scientists. The ability of each parameter to discriminate between high- and low-level performers as well as its interactions with other factors should be considered. Practitioners should also consider that parameters which differentiate athletes at one competition level may not be as valuable as athletes progress.

The aim of this study was to examine whether a contemporary TID testing battery successfully discriminated between high and lower performers in an elite junior swimming cohort. We utilised multiple regression models to examine the extent to which the parameters accounted for variation in competition performances across all competitive strokes. We also examined whether these relationships differ between sexes and event distances. The outcomes of this study should inform the choice and development of testing protocols used in elite junior swimmers.

\section{Materials and methods}

Forty-eight elite national-level junior swimmers (22 males, age $16.5 \pm 1.2 \mathrm{y}, 26$ females, age $15.5 \pm 1.1 \mathrm{y}$; mean \pm SD) completed a TID testing battery at the Australian Institute of Sport. This research was approved by the Australian Institute of Sport Research Ethics Committee.

A cross-sectional non-randomised design was used to assess physical and anthropometric parameters in accordance with the TID testing battery. This design was adopted given the logistical constraints of the testing period. Testing took place over four days in the morning prior to any swimming or dry-land training. The morning completion of maximal efforts would likely have limited any effects of circadian rhythm and training fatigue. Athletes were given the opportunity to eat prior to each morning testing session, although the content and timing of their meals were not recorded. The 400, 200 and $100 \mathrm{~m}$ steps of the CS test were conducted on days 1, 2 and 3 respectively, with jumps testing completed on day 4. Anthropometric testing took place on either day 3 or 4 , prior to any other testing or exercise, and after breakfast.

All measures were a part of a TID testing battery at the time this investigation occurred. The testing battery aimed to produce a holistic description of a swimmer's physical capabilities.

To calculate CS and supra-CS distance capacity (D') each swimmer completed a series of $400 \mathrm{~m}, 200 \mathrm{~m}$ and $100 \mathrm{~m}$ front-crawl maximal effort time trials on different days. Each time trial was completed in a long course $(50 \mathrm{~m})$ pool with a standard competition dive start and timed electronically using a touch pad system (Omega, Switzerland). Each time trial followed a standardised warm up (1700 m total, $400 \mathrm{~m}$ of race pace intensity including kick, pull and swim efforts) which concluded five min prior to the maximal effort. A linear regression relationship was then established for each individual swimmer $($ time $=\mathrm{x}$, distance $=\mathrm{y})$. The slope and the intercept terms of this line were then used as measures of CS and D' respectively. ${ }^{12}$
Athletes were measured for height, mass, sitting height, sum of 7 skinfolds, foot length, biacromial, biliocristal, transverse chest and femur breadths, and anterior-posterior chest depth using standardised methods. ${ }^{13}$ Assessment was undertaken by individuals accredited to a minimum of level two by the International Society for the Advancement of Kinanthropometry (ISAK). Date of birth was also recorded.

Sex, sitting height, height, mass and current age were used to calculate age at peak height velocity. ${ }^{14}$ Years from PHV was calculated as the difference between age at peak height velocity and current age (Eqs. (1) and (2)). A negative value indicated the swimmer was younger than PHV, a positive value indicated they were older.

(Male) :

$$
\begin{aligned}
& \text { PHV Offset }=-9.236+0.0002708 \times(\text { Leg } \times \text { Trunk }) \\
& -0.001663 \times(\text { Age } \times \text { Leg })+0.007216 \times(\text { Age } \times \text { Trunk }) \\
& +0.02292 \times\left(\frac{\text { mass }}{\text { height }} \times 100\right)
\end{aligned}
$$

(Female) :

PHV Offset $=-9.376+0.0001882 \times($ Leg $\times$ Trunk $)$

$$
\begin{aligned}
& +0.0022 \times(\text { Age } \times \text { Leg })+0.005841 \times(\text { Age } \times \text { Trunk }) \\
& -0.002658 \times(\text { Age } \times \text { Weight })+0.07693 \times\left(\frac{\text { mass }}{\text { height }} \times 100\right)
\end{aligned}
$$

where: Age = days between date of testing and date of birth/365.25, Trunk = measured sitting height $(\mathrm{cm})$, Height $=$ measured standing height $(\mathrm{cm})$, mass $=$ measured mass $(\mathrm{kg})$, Leg $=$ Height $-\operatorname{Trunk}(\mathrm{cm})$.

All swimmers were screened by a certified strength and conditioning coach to ascertain their strength training experience, and readiness to test. Eight swimmers with an injury or excessive fatigue were excluded from testing. The remaining athletes undertook six unloaded and three loaded countermovement jumps (10 kg, mass adjusted methodology of Kraska et al.). ${ }^{15}$ The unloaded jump test was performed after a 5-min dynamic warm-up, which included dynamic stretching, increasing intensity shuttle runs and body-weight squats followed by a series of jumps at progressively increasing intensities. Verbal instructions were provided for $50 \%$, $75 \%$ and $100 \%$ of maximal effort. After the unloaded jump protocol swimmers were familiarised with the loaded jump protocol. Familiarisation for the loaded jump protocol occurred at a verbally instructed intensity of $85 \%$ of maximal effort. Swimmers were then given $5 \mathrm{~min}$ of passive rest prior to testing the loaded condition. All jumps were separated by $3-5 \mathrm{~min}$ and the best jump, using jump height (typical error $2.4 \mathrm{~cm}$, coefficient of variation $5.2 \%)^{19}$ as the criterion parameter, was taken for analysis in both conditions. Ground reaction forces, jump height and velocity were measured using a combined force plate and position transducer system (Innervations Inc., Muncie, IN).

Swimming competition performances were sourced from publicly available websites. State and national sports association websites were searched for all state championship, national championship or state representative meet results within three months of the testing period. All competitions were short-course format. The duration between competition performance and the start of the testing period was $15 \pm 17$ days (mean \pm SD). Each swimmers' best time in each event at any of these competitions were recorded. Competition results in all strokes were divided by the current world record for their respective events to calculate a world record ratio (WRR) which was subsequently used as a standardised measure of performance (Eq. (3)). The event that had the lowest WRR was recorded as the athlete's best event and the stroke for this event deemed their best stroke. Where swimmers had $100 \mathrm{~m}$ and $200 \mathrm{~m}$ performances in their best stroke these were included in the 
distance-specific analyses. Swimmers were also categorised into $\leq 100 \mathrm{~m}$ or $\geq 200 \mathrm{~m}$ distance groups depending on their best event.

$\mathrm{WRR}=\frac{\text { time }_{\mathrm{i}}}{\text { world record }_{\mathrm{i}}} \times 100$

where time $e_{i}$ is a performance in $s$ in event $i$, and world record is the time in $\mathrm{s}$ for the same i event. World records were the current open age group short course records as of 1st of January 2016.

Descriptive statistics are reported as mean \pm SD. Pearson's correlations were used to analyse individual relationships between parameters. Multiple regression equation combinations were evaluated using the adjusted $\mathrm{R}^{2}$ measure. Multicollinearity of each component included in multiple linear regression models was assessed using variance inflation factor (VIF) values, with thresholds set at maximum of 10. For VIF interpretation the higher the value the greater the overall correlation the variable in question has with the rest of the explanatory variables. Normal distribution of parameters was assessed using the Shapiro-Wilk test. Heteroscedasticity was tested for using a Breusch-Pagan test and multivariate normality was assessed using Mardia's multivariate normality test. Fishers $r$ to $\mathrm{z}$ transformation tested differences in $r$ values when comparing multiple linear regression models (alpha $=0.05$ ).

Predicted performance changes were calculated by first fitting each multiple linear regression model using the mean values of each parameter for the group the model was created for. An increase of two standard deviations as per the methodology of Higham et al. ${ }^{16}$ was then applied to one parameter, and differences between model outcomes were then calculated and reported as the predicted performance change.

ANOVA analysis on distance group ( $<100 \mathrm{~m}$ or $>200 \mathrm{~m}$ specialists) and sex effects on all parameters were tested for normally distributed residuals using the Shapiro-Wilk test. Where these were not normally distributed an aligned rank transformation was used. Data were also checked for homogeneity of variance using a Levene's test. Tukey post-hoc analysis was performed for normally distributed data (alpha $=0.05$ ).

\section{Results}

For clarity, we have separated the results section into two parts, the first section details the results of the group mean analysis and the second section consists of the multiple linear regression analysis and describes the quantitative effect of selected parameters on the sub-groups of our sample.

Relevant variables that either had some influence on the overall interpretation of the data or were important for showing equivalence between sub-groups are presented in Table 1. There were significant differences between males and females in almost all parameters. Biiliocristal breadth was similar between sexes. Relative to the world record, competition performances were similar between sexes for $100 \mathrm{~m}$ and $200 \mathrm{~m}$ performances. The female group was approximately 1 year younger than the male group but were well matched for maturity (years from PHV).

There were two main outcomes from the multiple linear regression analysis. The first outcome was the models themselves, which are described in Table 2. The second outcome was the quantitative effect of each variable. To illustrate this a 2 SD change ${ }^{16}$ and the corresponding effect on performance for each variable for each model is shown in Table 3. Multiple linear regression models for males explained more variance than for the female swimmers. The models used a combination of anthropometry, maturation and performance parameters.

The male $100 \mathrm{~m}$ group had the most variance explained (88\%) using a combination of loaded jump height, years from PHV, humerus breadth, mass and D' (Table 2). Variance inflation factors were all $<5.0$, suggesting no overt issues with multi-collinearity. All parameters were positively associated with performance apart from mass, which was negatively correlated (Table 3 ).

Male $200 \mathrm{~m}$ performances were differentiated using broadly similar parameters as male $100 \mathrm{~m}$ performances. However, the differences between events included the incorporation of both loaded jump height and jump height in the $200 \mathrm{~m}$ model (all VIF values $<6.0$ ) and replacing D' with CS as a swimming performance indicator.

Female $100 \mathrm{~m}$ performances had marginally less total variance explained (74\%) when compared to male $100 \mathrm{~m}$ performances (Fishers $r$ to $z, p=0.09$ ). D' did not increase the accuracy of the model, however in the female cohort the chest depth and sitting height interaction explained a substantial proportion of variation.

Female $200 \mathrm{~m}$ performances were less related to physical or jump parameters than the $100 \mathrm{~m}$ performances in this investigation (Fishers $r$ to $z, p=0.02$ ). Larger chest depths and faster CS values were related to improved performance. In contrast to the male cohort where we observed a positive effect of maturity on performance, there was little explanatory value for years from PHV in our female cohort.

\section{Discussion}

In this investigation we cross-sectionally differentiated between performances relative to the world record for male and female elite junior swimmers using selected TID testing parameters. These distinctions were across all strokes and especially effective for $100 \mathrm{~m}$ performances. Overall, it appears that more powerful, more mature and lighter males, and more powerful females with a larger chest depth and shorter sitting heights, had a competitive advantage in $100 \mathrm{~m}$ events regardless of stroke. These insights may assist coaches when selecting talented junior swimmers.

The TID testing parameters correlated more closely with $100 \mathrm{~m}$ performances than $200 \mathrm{~m}$ performances. The female $200 \mathrm{~m}$ group had the lowest variance in performance explained (33\%) by the TID testing parameters, suggesting that most of the variation in this group relates to parameters outside our measures. The female $100 \mathrm{~m}$ group had a greater amount of variance explained (74\%), largely in relation to the chest depth:sitting height interaction. The interaction of these parameters may partly explain contrasting findings on the effect of sitting height on performance. We observed that a larger sitting height was detrimental to performance when chest depth was kept constant. These variables were not highly correlated (Adjusted $\mathrm{R}^{2}=0.13$ ). This outcome contrasts earlier reports ${ }^{5}$ that a larger sitting height was beneficial for performance. These differences could relate to differences in analyses or the higher performance level in our investigation (mean WRR $=113.3$ compared to 125.1 ).

Our multiple regression models also showed that selected anthropometric parameters did not relate as closely to performance in our cohort compared to previous research. These studies indicate substantial associations between height ${ }^{17-19}$ and $\operatorname{arm~span~}^{5,20}$ for males and females. It is important to note that our models aimed to describe the best combination of parameters to explain variance in performances. Therefore, parameters such as height and arm span which were highly correlated with parameters (in this case sitting height) which were included in the models were not utilised to avoid multi-collinearity.

In the female cohort, the TID testing parameters were less associated with performance than the males, agreeing with some previous research ${ }^{5}$ and in contrast with other investigations. ${ }^{17}$ Relative lower limb power was related to $100 \mathrm{~m}$ performances in combination with sitting height and chest depth, however rela- 
Table 1

Differences in competitive performances, anthropometry, critical speed parameters and predicted maturity between sexes and event distance.

\begin{tabular}{|c|c|c|c|c|c|}
\hline \multicolumn{6}{|l|}{ Sex } \\
\hline Parameter & Male & Female & Difference & $95 \%$ confidence limits $( \pm)$ & $\mathrm{p}$ Value \\
\hline Critical speed $\left(\mathrm{m} \mathrm{s}^{-1}\right)$ & 1.45 & 1.32 & 0.12 & 0.03 & $<0.001$ \\
\hline $\mathrm{D}^{\prime}(\mathrm{m})$ & 20.7 & 19.4 & 1.3 & 2.1 & 0.21 \\
\hline Years from PHV (y) & 3.2 & 3.2 & 0.0 & 0.5 & 0.88 \\
\hline Age at PHV $(y)$ & 13.3 & 12.3 & 1.1 & 0.3 & $<0.001$ \\
\hline Current age (y) & 16.5 & 15.5 & 1.0 & 0.7 & $<0.01$ \\
\hline WRR $100 \mathrm{~m}(\%)$ & 114.9 & 113.2 & 1.7 & 2.1 & 0.11 \\
\hline WRR 200 m (\%) & 113.0 & 112.7 & 0.4 & 1.9 & 0.70 \\
\hline
\end{tabular}

Distance group

\begin{tabular}{|c|c|c|c|c|c|}
\hline Parameter & $100 \mathrm{~m}$ & $200 \mathrm{~m}$ & Difference & $95 \%$ confidence limits $( \pm)$ & $\mathrm{p}$ Value \\
\hline Height $(\mathrm{cm})$ & 177.9 & 176.9 & -1.3 & 3.3 & 0.03 \\
\hline Sum of 7 skinfolds (mm) & 62.9 & 68.9 & 6.0 & 8.2 & $<0.01$ \\
\hline Critical speed $\left(\mathrm{m} \mathrm{s}^{-1}\right)$ & 1.33 & 1.41 & 0.08 & 0.03 & $<0.01$ \\
\hline $\mathrm{D}^{\prime}(\mathrm{m})$ & 22.5 & 18.3 & -4.2 & 2.1 & $<0.001$ \\
\hline Years from PHV (y) & 3.6 & 3.0 & -0.6 & 0.6 & $<0.01$ \\
\hline Age at $\operatorname{PHV}(y)$ & 12.7 & 12.8 & -0.1 & 0.3 & 0.72 \\
\hline Current age (y) & 16.2 & 15.7 & -0.5 & 0.7 & 0.03 \\
\hline WRR $100 \mathrm{~m}(\%)$ & 112.5 & 115.0 & 2.5 & 2.1 & 0.02 \\
\hline WRR $200 \mathrm{~m}(\%)$ & 114.0 & 112.3 & -1.8 & 2.1 & 0.09 \\
\hline
\end{tabular}

Male distance group

\begin{tabular}{|c|c|c|c|c|c|}
\hline Parameter & $100 \mathrm{~m}$ & $200 \mathrm{~m}$ & Difference & $95 \%$ confidence limits $( \pm)$ & p Value \\
\hline$D^{\prime}(m)$ & 24.4 & 19.3 & -5.1 & 4.6 & 0.02 \\
\hline
\end{tabular}

Female distance group

\begin{tabular}{|c|c|c|c|c|c|}
\hline Parameter & $100 \mathrm{~m}$ & $200 \mathrm{~m}$ & Difference & $95 \%$ confidence limits $( \pm)$ & p Value \\
\hline Sum of 7 skinfolds (mm) & 69.1 & 87.9 & 18.9 & 14.4 & 0.01 \\
\hline $\mathrm{D}^{\prime}(\mathrm{m})$ & 21.6 & 17.1 & -4.5 & 3.7 & 0.01 \\
\hline Years from PHV (y) & 3.6 & 2.9 & -1.1 & 1.0 & 0.03 \\
\hline
\end{tabular}

Table 2

Final multiple regression models for estimating male and female 100 and $200 \mathrm{~m}$ World Record Ratios (WRRs)

\begin{tabular}{|c|c|c|c|c|c|c|c|c|c|c|}
\hline Group & $\mathrm{n}$ & $\mathrm{r}^{2}$ & Adj $r^{2}$ & $\mathrm{p}$ & VIF 1 & VIF2 & VIF3 & VIF4 & VIF5 & $\begin{array}{l}\text { Residual standard error } \\
\text { (degrees of freedom) }\end{array}$ \\
\hline \multirow[t]{2}{*}{ Male } & \multicolumn{10}{|c|}{$100 \mathrm{~m}$ WRR $=-1.10 \times($ loaded jump height $)-5.15 \times($ humerus breadth $)+0.35 \times($ mass $)-0.31 \times\left(D^{\prime}\right)-1.53 \times($ years from PHV $)+172.12$} \\
\hline & 16 & 0.93 & 0.88 & $<0.001$ & 1.72 & 1.72 & 4.84 & 1.31 & 3.85 & $1.52(7)$ \\
\hline \multirow[t]{2}{*}{ Male } & \multicolumn{10}{|c|}{$\begin{array}{l}200 \mathrm{~m} \text { WRR }=-1.95 \times \text { (years from PHV })+4.90 \times \text { (loaded jump height })+2.16 \times(\text { mass })-0.48 \times(\text { jump height })-48.96 \times(C S)-0.06 \times(\text { loaded jump } \\
\text { height } x \text { mass })+48.47\end{array}$} \\
\hline & 20 & 0.84 & 0.73 & 0.003 & $4.30^{*}$ & $5.52^{*}$ & $4.36^{*}$ & $2.42^{*}$ & $3.45^{*}$ & $1.88(9)$ \\
\hline \multirow[t]{2}{*}{ Female } & \multicolumn{10}{|c|}{$100 \mathrm{~m}$ WRR $=-10.00 \times($ sitting height $)-56.44 \times($ chest depth $)+0.60 \times($ loaded jump height $)+0.61 \times($ chest depth $\times$ sitting height $)+1054.94$} \\
\hline & 21 & 0.82 & 0.74 & 0.002 & $1.10^{*}$ & $1.27^{*}$ & $1.17^{*}$ & & & $1.55(9)$ \\
\hline \multirow[t]{2}{*}{ Female } & \multicolumn{10}{|c|}{$200 \mathrm{~m}$ WRR $=-192.19 \times(\mathrm{CS})-14.33 \times($ chest depth $)+10.56 \times($ CS $\times$ chest depth $)+372.42$} \\
\hline & 19 & 0.44 & 0.33 & 0.03 & 1.07 & 1.07 & & & & $1.90(15)$ \\
\hline
\end{tabular}

${ }^{*}$ VIF values calculated excluding interaction effects.

tive leg power did not relate strongly to $200 \mathrm{~m}$ performance. It seems that female $200 \mathrm{~m}$ performances can be partly explained by the level of aerobic fitness indicated by CS. It may be that technical proficiency relates more closely to female than male $200 \mathrm{~m}$ performances. ${ }^{5}$ Technical proficiency tests, such as the $6 \times 50 \mathrm{~m}$ stroke efficiency test, ${ }^{1}$ were not a part of the TID testing battery and could be explored in future research.

Larger participant numbers and greater variation in performance level would have permitted clearer, more detailed results. The 3-month time frame for performances in relation to testing was also a limitation, although most athletes' performances (31 of 48 ) were within 4 days of testing. If the competition results for the remaining 17 athletes had been closer to the testing period this may have led to clearer results. This shortcoming does not apply to all parameters however as some, such as height, vary little over a 3-month period even in adolescent athletes.

Practically the results of our analysis indicate that current TID testing parameters appear more appropriate for male, rather than female athletes. It appears there is justification for introducing some new tests for female athletes that are more aligned with competitive performance. New tests for female athletes could focus on technical aspects, rather than physiology, such as the $6 \times 50 \mathrm{~m}$ stroke efficiency test. ${ }^{1}$ Furthermore, the testing protocols better align to performance in $100 \mathrm{~m}$ than $200 \mathrm{~m}$ swimming events. The predictive models estimate improvements related to trainable measures while controlling for important individual characteristics that are less influenced by environmental factors. For male participants, these models also provide insight in to the perennial question on the relative merits of training to increase muscle hypertrophy and/or strength/power characteristics, in light of the potential costs of increasing swimmer mass and related drag. The male $100 \mathrm{~m}$ model indicates a change of $>0.3 \mathrm{~cm}$ in loaded jump performance per $\mathrm{kg}$ of muscle gained should improve swimming performance if all other parameters remain constant. Future research should examine whether a similar relationship is evident between body mass and upper body power measures. 
Table 3

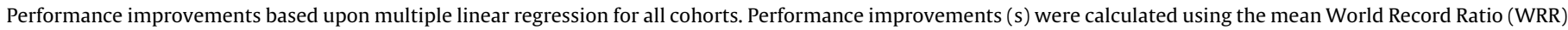
for each cohort converted to a performance time using the corresponding short course front-crawl world record. Negative values indicate a performance improvement.

\begin{tabular}{|c|c|c|c|c|c|}
\hline Parameter & $\mathrm{r}$ & $2 \mathrm{SD}$ & $\begin{array}{l}\text { Performance } \\
\text { improvement (\% points) }\end{array}$ & $\begin{array}{l}\text { Performance } \\
\text { improvement (s) }\end{array}$ & $\begin{array}{l}\text { Mean performance } \\
\text { time (WRR \& time) }\end{array}$ \\
\hline \multicolumn{6}{|l|}{ Male $100 \mathrm{~m}$} \\
\hline Loaded jump height (cm) & -0.79 & 6.9 & -7.6 & -3.9 & $115 \%$ \\
\hline Years from PHV $(\mathrm{y})$ & -0.39 & 1.9 & -3.0 & -1.5 & 51.62 \\
\hline Mass (kg) & -0.55 & 14.8 & 5.2 & 2.7 & \\
\hline Humerus breadth (mm) & -0.41 & 0.7 & -3.6 & -1.9 & \\
\hline $\mathrm{D}^{\prime}(\mathrm{m})$ & -0.44 & 7.5 & -2.3 & -1.2 & \\
\hline \multicolumn{6}{|l|}{ Male $200 \mathrm{~m}$} \\
\hline Loaded jump height (cm) & -0.47 & 10.9 & 0.3 & 0.4 & $113 \%$ \\
\hline Years from PHV $(y)$ & -0.39 & 2.0 & -3.9 & -4.4 & $1: 52.32$ \\
\hline Mass $(\mathrm{kg})$ & -0.35 & 14.5 & 2.2 & 2.4 & \\
\hline Jump height $(\mathrm{cm})$ & -0.55 & 8.5 & -4.1 & -4.6 & \\
\hline Critical speed $\left(\mathrm{m} \mathrm{s}^{-1}\right)$ & -0.42 & 0.1 & -5.1 & -5.7 & \\
\hline \multicolumn{6}{|l|}{ Female $100 \mathrm{~m}$} \\
\hline Breadth AP chest depth $(\mathrm{cm})$ & $-0.42 \quad 3.6$ & & -4.3 & -2.5 & $113 \%$ \\
\hline Sitting height $(\mathrm{cm})$ & $-0.50 \quad 5.9$ & & 2.2 & 1.3 & 57.73 \\
\hline Loaded jump height $(\mathrm{cm})$ & $-0.39 \quad 7.1$ & & -4.3 & -2.5 & \\
\hline \multicolumn{6}{|l|}{ Female $200 \mathrm{~m}$} \\
\hline Critical speed $\left(\mathrm{m} \mathrm{s}^{-1}\right)$ & -0.47 & 0.1 & -1.3 & -1.7 & $112 \%$ \\
\hline Breadth AP chest depth $(\mathrm{cm})$ & -0.44 & 3.6 & -1.5 & -1.8 & $2: 04.72$ \\
\hline
\end{tabular}

\section{Conclusion}

The results of our investigation suggest that for sprint-based male swimmers that loaded jump height, humerus breadth, mass, D' and years from PHV should be included for TID testing in the future. For female sprinters sitting height, chest depth and loaded jump height are the most important variables to include. For $200 \mathrm{~m}$ swimmers critical speed was an important parameter, but anthropometry and lower limb power parameters were less important. To refine this testing battery the addition of a test specifically for technical proficiency, which was not assessed in this investigation, may be of benefit; particularly for swimmers who specialise in longer events where efficiency may be more important.

\section{Practical applications}

- When applying TID testing for adolescent male swimmers practitioners should include some indication of maturity status.

- Loaded jump height was the most useful metric from lower limb power testing and should be prioritised when testing the physical capacities of junior swimmers of both sexes.

- For female swimmers, chest depth seems to be a particularly important anthropometrical parameter.

- Further refinement of TID testing protocols is required to explain more variation in performance between swimmers, particularly for female $200 \mathrm{~m}$ swimmers.

\section{Acknowledgements}

The authors would like to acknowledge the work of all the staff and students at the Australian Institute of Sport who contributed to the collection of this data. Without them this investigation would not have been possible.

\section{References}

1. Tanner R, Gore C. Physiological Tests for Elite Athletes, 2nd ed. Lower Mitcham, South Australia, Human Kinetics, 2013.

2. 2016 Junior Pan Pacific Championship Athlete Selection Criteria. swimming.org. au. doi:https://doi.org/10.1109/panpacific.2016.7428384.
3. Barbosa TM, Costa MJ, Marinho DA. Proposal of a deterministic model to explain swimming performance. Int J Swim Kin 2013; 2(1):1-54

4. Carter J, Ackland TR. Kinanthropometry in Aquatic Sports: A Study of World Class Athletes (HK Sport Science Monograph Series 5), Champaign (IL), Human Kinetics, 1994.

5. Silva AJ, Costa AM, Oliveira PM et al. The use of neural network technology to model swimming performance. J Sports Sci Med 2007; 6(1):117-125.

6. Strzala M, Tyka A. Physical endurance, somatic indices and swimming technique parameters as determinants of front crawl swimming speed at short distances in young swimmers. Med Sport 2009; 13(2):99-107.

7. Capelli C, Pendergast DR, Termin B. Energetics of swimming at maximal speeds in humans. Eur J Appl Physiol 1998; 78(5):1-9. http://dx.doi.org/10.1007/s004210050435.

8. Rodriguez F, Mader A. Energy metabolism during 400 and 100-m crawl swimming: computer simulation based on free swimming measurement. Biomech Med Swim IX 2003:373-378.

9. West DJ, Owen NJ, Cunningham DJ et al. Strength and power predictors of swimming starts in international sprint swimmers. J Strength Cond Res 2011; 25(4):950-955.

10. Cossor J, Mason B. Swim start performances at the Sydney 2000 Olympic Games. ISBS-Conf Proc Arch 2001; 1(1).

11. Baxter-Jones A, Helms P. Born too late to win? Nature 1994; 370(6486):186. http://dx.doi.org/10.1038/370186b0.

12. Wakayoshi K, Yoshida T, Udo M et al. Does critical swimming velocity represent exercise intensity at maximal lactate steady state? Eur J Appl Physiol 1993: 66(1):90-95.

13. Stewart AD, Marfell-Jones MJ, Olds TS et al. International Standards for Anthropometric Assessment, New Zealand, International Society of the Advancement of Kinanthropometry, 2011.

14. Mirwald RL, Baxter-Jones AD, Bailey DA et al. An assessment of maturity from anthropometric measurements. Med Sci Sports Exerc 2002; 34(4):689-694.

15. Kraska JM, Ramsey MW, Haff GG et al. Relationship between strength characteristics and unweighted and weighted vertical jump height. Int J Sports Physiol Perform 2009; 4(4):461-473.

16. Higham DG, Hopkins WG, Pyne DB et al. Performance indicators related to points scoring and winning in international rugby sevens. J Sports Sci Med 2014; 13(2):358-364 https://www.ncbi.nlm.nih.gov/pubmed/24790490.

17. Siders WA, Lukaski HC, Bolonchuk WW. Relationships among swimming performance, body composition and somatotype in competitive collegiate swimmers. J Sports Med Phys Fitness 1993; 33(2):166-171.

18. Jurimae J, Haljaste K, Cicchella A et al. Analysis of swimming performance from physical, physiological, and biomechanical parameters in young swimmers. Pediatr Exerc Sci 2007; 19(1):70-81.

19. Huijing PA, Toussaint HM, Mackay R et al.Active Drag Related to Body Dimensions, In: Ungerechts B, Wilke K, Reis-chle K, editors, Champaign, Illinois, Swimming Science V, 1988, p. 31-37.

20. Lätt E, Jürimäe J, Mäestu J et al. Physiological, biomechanical and anthropometrical predictors of sprint swimming performance in adolescent swimmers. J Sport Sci Med 2010; 9(3):398. 